\title{
Displasia osteofibrosa de cornete inferior: causa inusual de obstrucción nasal
}

\author{
Fibous dysplasia of the inferior turbinate: \\ an unusual cause of nasal obstruction
}

\author{
Christian Olavarría $L^{1}$, Michel Royer $\mathrm{F}^{1}$, Fernando Insunza $\mathrm{P}^{2}$, María Elena Villanueva $\mathrm{I}^{3}$, \\ Constanza Valdés $\mathrm{P}^{1}$.
}

\begin{abstract}
RESUMEN
La displasia osteofibrosa es una patología ósea benigna e idiopática, poco frecuente en cabeza y cuello. Afecta principalmente el hueso maxilar, mientras en fosanasal su frecuencia es baja Habitualmente asintomática, puede ser agresiva por crecimiento local y eventual malignización. $\theta$ tratamiento es quirúrgico cuando produce alteraciones en la estética facial, síntomas compresivos orbitarios, rinosinusales, vasculares, nerviosos o ante sospecha de malignización. Se presenta un caso de un paciente con obstrucción nasal persistente, en cuya evaluación se diagnostica un tumor óseo de cornete inferior, que es extirpado mediante técnica endoscópica con resultado satisfactorio. 1 estudio histopatológico confirmó el diagnóstico de displasia osteofibrosa Se discuten los problemas diagnósticos y terapéuticos, destacando la importancia del examen físico y el diagnóstico oportuno, pudiendo tratarse endoscópicamente sin inconvenientes cuando son pequeños.
\end{abstract}

Palabras clave: Displasia osteofibrosa, Cornete inferior, Tumores benignos de cabeza y cuello.

\section{ABSTRACT}

Fibrous dysplasia is an idiopathic benign bone disease, infrequent in head and neck. It is mainly located in the maxillary bone, while its nasal cavity frequency is low. It is usually asymptomatic, but it can be aggressive due to local growth and malignant degeneration. Its treatment is surgical when the disease produces an important facial deformity, orbital, rhinosinusal or vascular-nervous compressive symptoms or when malignization is suspected. A case report of a patient with persistent nasal obstruction is presented. Upon evaluation, a bony tumor of the inferior turbinate was diagnosed. Excision was performed using endoscopic technique, with satisfactory clinical results. Histopathological examination confirmed the diagnosis of fibrous dysplasia We emphasize the importance of the otolaryngology physical examination and the early diagnosis in order to offer the more adequate treatment when lesions are small, so as to be able to perform an endoscopic surgery without potential disadvantages.

Key words: Fbrous dysplasia, inferior turbinate, head and neck benign tumors.

\footnotetext{
'Médico Servicio de Otorrinolaringología, Hospital Gínico de la Universidad de Chile. Médico Otorrinolaringólogo, Hospital Las Higueras, Talcahuano.

3Médico Servicio de Anatomía Patológica, Hospital Cínico de la Universidad de Chile.
} 


\section{INTRODUCCIÓN}

La displasia osteofibrosa (DOF) es una patología ósea benigna e idiopática ${ }^{1-6}$ del esqueleto, que se caracteriza por el remplazo de la médula ósea por tejido fibroso y óseo desorganizado2,4,7,8. Su frecuencia en cabeza y cuello es baja, representando el $2 \%$ de todas las neoplasias óseas. Afecta de forma más frecuente el hueso maxilar, frontal, mandíbula, parietal y occipital; en fosa nasal su incidencia es baja ${ }^{1,2}$. Es más frecuente en el sexo femenino, alrededor de la segunda década de la vida ${ }^{3,5}$; existiendo dos formas clínicas: la displasia monostótica, que compromete un sólo hueso y constituye el $80 \%$ de los casos, y la poliostótica que compromete más de un hueso ${ }^{1-5,7,8}$. La forma poliostótica presenta un subtipo, el síndrome de McOune-Albright, que se caracteriza por la tríada de pubertad precoz periférica, manchas cutá neas café con leche de bordes irregulares y displasia poliostótica-4,8,9.

La DOF es una patología benigna frecuentemente asintomática y cuya evolución finaliza en la pubertad, pero debe recordarse que puede ser agresiva por su crecimiento local y tiene riesgo de malignización a osteosarcoma o fibrosarcoma?

日 objetivo de este trabajo es aportar con un caso clínico de obstrucción nasal secundaria a DOF de cornete inferior y discutir los aspectos diagnósticos y terapéuticos de esta patología.

\section{CASO CLÍNICO}

Se presenta el caso de un paciente de sexo masculino, 16 años, sin antecedentes mórbidos relevantes, con un cuadro de aproximadamente 2 años de evolución caracterizado por obstrucción nasal, principalmente en fosa nasal izquierda. Evaluado en primera instancia por medicina general, refiere prurito nasal, rinorrea serosa y antecedentes de atopia familiar (rinitis alérgica y asma) por lo que se inicia tratamiento con antihistamínicos. Se controla en una segunda oportunidad con pobre respuesta al tratamiento por lo que se asocian esteroides nasales. Posteriormente, debido a su tórpida evolución, se solicitan recuento de eosinó- filos nasales y test cutáneo a aeroalergenos, los que resultan concordantes con alergia a pastos/ gramíneas. En este contexto se enfatizan medidas ambientales, se aumentan dosis de esteroides nasales y se logra una buena adherencia del paciente al tratamiento. No obstante, pese a la disminución de síntomas pruriginosos, persiste la obstrucción nasal, por lo que se decide enviar al otorrinolaringólogo, en cuya evaluación destaca una mucosa levemente edematosa, sin rinorrea y una hipertrofia de cornete inferior unilateral, con consistencia dura a la palpación. Se solicita una tomografía computada de cavidades perinasales en la que destaca un aumento de volumen óseo del cornete inferior izquierdo de 5 por 6 centímetros, sin compromiso de vía lagrimal y velamiento del seno maxilar ipsilateral determinado por una obstrucción parcial del drenaje (Figura 1).

La lesión se extrajo por vía endoscópica bajo anestesia general, en forma submucosa confeccionando un flap en el corneteinferior izquierdo, resección del tumor siguiendo un plano de clivaje mediante fresa y cizalla. Se optimizó el drenaje del seno maxilar mediante uncinectomía y antrostomía, encontrándose secreción y mucosa engrosada. Dado que los hallazgos operatorios fueron concordantes con la extensión tomográfica no fue necesario el abordaje de etmoides. La lesión fue extraída sin dañar las estructuras vecinas y se repuso el flap de mucosa sobre el cornete inferior. $\boxminus$ análisis histológico demostró trabéculas arciformes, sin osteoblastos en la periferia y con estroma fusado laxo entre ellas, por lo que se diagnosticó displasia osteofibrosa (Fgura2).

La evolución posoperatoria fue satisfactoria con disminución significativa de la sintomatología obstructivay en el seguimiento se hamantenido sin evidencias de recidiva, tanto clínica como radiológica

\section{DISCUSIÓN}

La displasia osteofibrosa es una patología benigna, inicialmente asintomática, cuya presentación clínica depende de su ubicación, tamaño y crecimiento. desarrollo en fosa nasal tiene una baja frecuencia, siendo descrita previamente en cornete medio $0^{1,7,10}$. 


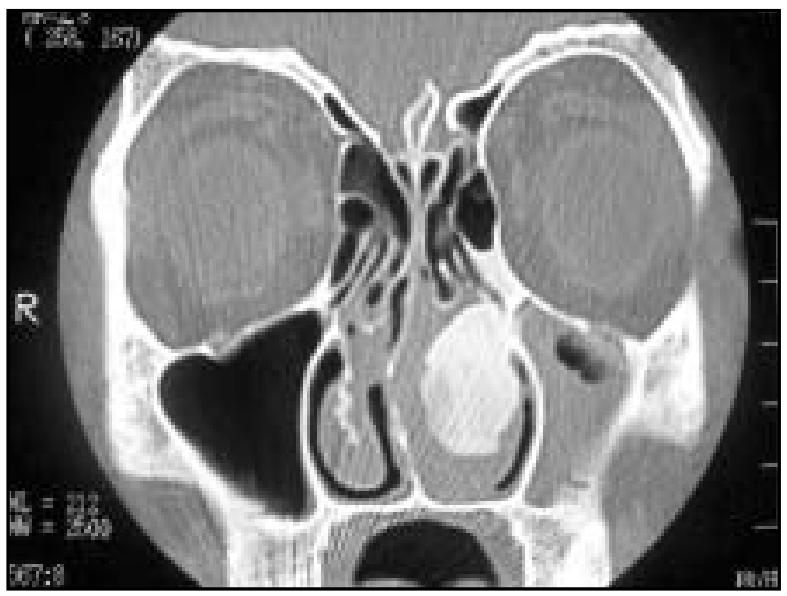

Figura 1. Tomografía computada de cavidades paranasales que demuestra un aumento de volumen óseo dependiente del cornete inferior izquierdo, sin compromiso de la vía lagrimal y velamiento parcial del seno maxilar ipsilateral.

Figura2. Corte histológico de tumor extraído. Destaca la presencia de trabéculas óseas arciformes (a), estroma fusado laxo (b) y ausencia de osteoblastos en la periferia (c), hallazgos concordantes con displasia osteofibrosa.

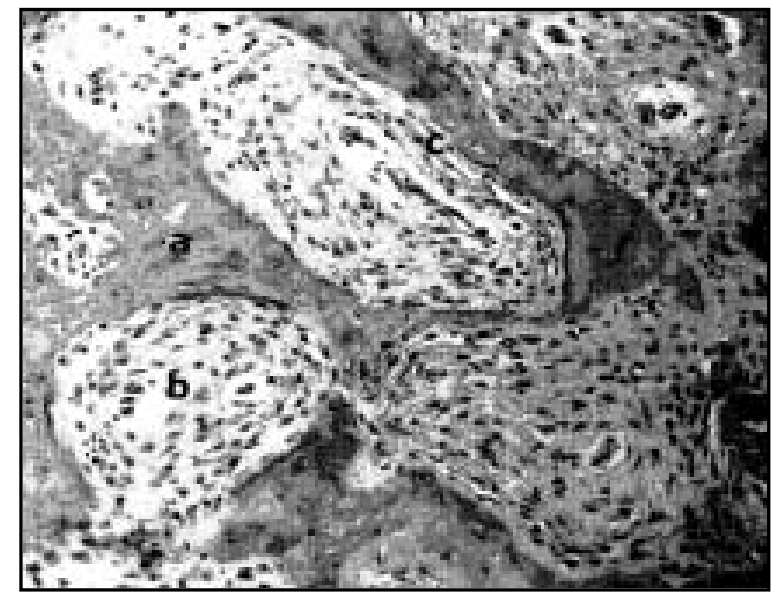

En el presente caso el paciente presentaba obstrucción nasal persistente, mientras otras formas de presentación pueden ser compresión de estructuras vecinas, atrapamiento de estructuras neurológicas 0 dolor óseo por fracturas ${ }^{1-3,7}$. Cabe destacar el lapso de tiempo en que el paciente fue tratado como rinitis alérgica, aproximadamente durante dos años, dado que presentaba características clínicas y exámenes compatibles con esta enfermedad. Debe recordarse que en patologías rinosinusales el examen endonasal es la primera herramienta diagnóstica y no puede ser reemplazada por otro tipo de evalua ciones. La rinoscopía anterior nos permite evaluar las fosas nasales y evidenciar alteraciones anatómicas significativas. Esto es necesario sobretodo en casos como el descrito, en los cuales los síntomas son predominantemente unilaterales y no existe una respuesta clínica adecuada, pese al óptimo tratamiento médico.

Como herramientas diagnósticas complementarias la tomografía computada de cavidades paranasales es muy específica en esta patología Presenta habitualmente tres patrones: pseudoquístico o insuflante (el más frecuente), esclerótico y el pagetoide ${ }^{1,4}$. Este examen permite valorar la extensión, su posible crecimiento y en los casos de tratamiento quirúrgico otorga valiosa información sobre la anatomía

Entre los diagnósticos diferenciales más significativos se consideran el fibroma osificante, osteomas y condromas. Dado que la presentación 
clínica no difiere significativamente entre ellos, es la correlación del aspecto imageneológico con el estudio histopatológico lo que finalmente otorgará el diagnóstico. Las características al estudio histológico son trabéculas óseas desorganizadas rodeadas por tejido fibroso denso, con ausencia de osteoblastos en la periferia trabecular ${ }^{1,2,6,8}$.

日 tratamiento en los pacientes con DOF debe ser indicado caso a caso dependiendo de la edad y estado del paciente, huesos comprometidos, síntomas asociados y sospecha de malignización. En general es quirúrgico, pudiendo ser mediante un abordaje externo o endoscópico, pero las recidivas son frecuentes $(10-25 \%)^{7,8,11}$. En este caso se decidió realizar la cirugía en forma endoscópica dada su menor morbilidad y capacidad de preservar la mucosa nasal no comprometida mediante una resección submucosa. Dentro de los desafíos quirúrgicos que se enfrentaron destacan el plano de clivaje, la alta consistencia y el gran tamaño del tumor. $日$ plano de clivaje pudo identificarse satisfactoriamente sin necesidad de explorar estructuras vecinas no comprometidas. Por su dureza se utilizaron cinceles, fresa y cizalla para la resección del tumor; el gran tamaño dificultaba su extracción requiriendo que se realizara una septoplastía, luxar el septum nasal para aumentando el campo operatorio y una partición endonasal del tumor para retirarlo por fosa nasal.

En conclusión debe destacarse que frente a un paciente con sintomatología de obstrucción nasal persistente siempre se debe realizar un examen otorrinolaringológico completo, se deben tener presentes diagnósticos diferenciales, existiendo patologías que pueden ser evidentes alarinoscopía anterior, siendo necesario su diagnóstico oportuno para otorgar el mejor tratamiento posible, dado que la vía de abordaje depende en buena medida del tamaño y extensión del tumor.

\section{BIBLIOGRAFÍA}

1. Alba J, Armengol M, Pérez C et al. Una FormaExcepcional de displasia fibrosa craneofacial: displasia fibrosa de cornete medio. Acta Aorrinolaringol Esp 2002; 53: 291-4.

2. OAVARRía C, CEEDÓN C, INZUNZA F, DE CAROLIS V, LeMP M. Displasia Osteofibrosa: Experiencia de 10 años. Rev Otorrinolaringol Cr Cabeza Quello 2003; 63: 55-62.

3. ROJAS R, PaLaGoO E KaPLAN J, Wong L. Fbrous dysplasia of the frontal sinus. Ear, Nose \& Throat J 2004; 83(1): 14-5.

4. CHANE Ethmoid fibrous dysplasia with anterior skull base and intraorbital extension. Ear, Nose \& Throat J 2005; 84(10): 627-8.

5. BORDAGARAY M, BordagaRAY P. Displasia fibrosa ósea. Rev Otorrinolaringol Cir Cabeza Quello 1997; 57: 141-8.

6. Papadakis C, SKOUlakis C, Prokopakis E et al. Fibrous dysplasia of the temporal bone: report of a case and a review of its characteristics. Ear, Nose \& Throat J 2000; 79(1): 52-7.

7. SaetT R, Silvestrini M, Marino F, NaRne S. Fibrous dysplasia of middle turbinate associated with Widal syndrome: endoscopic treatment of a rare case. Acta Atorhinolaryngol Ital 2004; 24(5): 288-91.

8. NeSON B, THOMPSON L. Fibrous dysplasia of bone. Ear, Nose \& Throat J 2003; 82(4): 259.

9. ROMAN R, JoNSON M, CORDNER D ET AL. Estudio clínico-molecular de pacientes chilenas con síndrome de Mcaune-Albright. Rev Méd Chile 2001; 129 (12): 1365-72.

10. LaBagnaRA J, YUPPA F, Klen MH Et AL. Fibrous dysplasia of the middle turbinate with primary hiperparatyoidism. Ear, Nose \& Throat J 1998; 77: 552-3.

11. Frgusan B. Fibrous dysplasia of the paranasal sinuses. Am J Oolaryngol 1994; 15(3): 227-34.

Dirección: Dr. Christian Oavarría Leiva

Santos Dumont 999. Independencia

Fax: 7378088

Email: christian.olavarria@gmail.com 